\title{
Aggregation dynamics of charged peptides in water: effect of salt concentration
}

Susmita Ghosh, ${ }^{1,2, a)}$ Devanand T, 1,2, b) Upayan Baul, ${ }^{3, \text { c) }}$ and Satyavani Vemparala ${ }^{1,2, d)}$

1) The Institute of Mathematical Sciences, C.I.T. Campus, Taramani, Chennai 600113, India

${ }^{2)}$ Homi Bhabha National Institute, Training School Complex, Anushakti Nagar, Mumbai, 400094, India

3) Institue of Physics, Albert-Ludwigs-University of Freiburg, Hermann-Herder-Strasse 3, 79104 Freiburg, Germany

(Dated: 26 April 2019)

Extensive molecular dynamics simulations have been employed to probe the effects of salts on the kinetics and dynamics of early-stage aggregated structures of steric zipper peptides in water. The simulations reveal that the chemical identity and valency of cation in the salt play a crucial roles in aggregate morphology of the peptides. Sodium ions induce the most aggregated structures but this is not replicated by potassium ions which are also monovalent. Divalent Magnesium ions induce aggregation, but to a lesser extent than that of sodium and their interactions with the charged peptides are also significantly different. The aggregate morphology in the presence of monovalent sodium ions is a compact structure with interpenetrating peptides, which differs from the more loosely connected peptides in the presence of either potassium or magnesium ions. The different ways in which the cations effectively renormalize the charges of peptides is suggested to be the cause of the differential effects of different salts studied here. These simulations underscore the importance of understanding both the valency and nature of of salts in biologically relevant aggregated structures.

\section{INTRODUCTION}

The process of aggregation of proteins in solution has been implicated in a wide range of diseases including Type II diabetes, Alzheimer's, Parkinson's and Huntington's disease $\mathrm{e}^{1,2}$. Understanding the underlying causes for the formation of aggregates of proteins, which are otherwise individually solvable, is far from complete. Given the important role of such protein aggregates in the aforementioned neurodegenerative diseases, it is also crucial to examine the role played by solvent conditions, including $\mathrm{pH}$, temperature, salt content etc., on promotion or degradation of such protein aggregates ${ }^{3-5}$. Much of the focus in protein aggregation studies has been on the formation of fibrillar structures, like in the case of amyloid aggregates, since they are directly related to diseases $^{6,7}$. Proteins, on the other hand, are also known to form amorphous aggregates which may lack precise structural information, but are nevertheless not the desired end product for protein solubility and protein functional aspects ${ }^{8-11}$.

The phase separation behaviour of proteins is strongly linked to the protein sequence along with the solution conditions, and is driven by complicated balance of interprotein, intra-protein, protein-solvent, solvent-solvent interactions along with the entropic contributions. Notably, proteins containing the so called "low-complexity regions" (LCRs) have the propensity to form condensates

\footnotetext{
a) Electronic mail: susmitag@imsc.res.in

b) Electronic mail: devanandt@imsc.res.in

c) Electronic mail: upayan.baul@physik.uni-freiburg.de

d) Electronic mail: vani@imsc.res.in
}

and the propensity is modulated by conditions such as temperature and ionic strength as reviewed in the past $^{12,13}$. Transitions from individually collapsed states to aggregate phase of LCRs are mainly demarcated by hydrophobicity, aromatic and aliphatic character, and charge content in low-complexity sequences ${ }^{14-22}$. The phase transition of proteins enriched with hydrophobic amino acids is likely to be mediated by hydrophobic interaction under physiological conditions and they are aggregation prone in general ${ }^{23}$. In contrast, LCRs with charged residues would result in high solubility and might prevent aggregation due to unfavorable electrostatic interaction between charged residues ${ }^{24,25}$. Understanding the molecular basis of such underlying phase transitions of charged LCRs at varying physiologically conditions is of particular interest.

Aggregation behavior of charged proteins/peptides considered as charged biopolymers, having a coarsegraining description have been studied rigorously before $^{26-41}$. In such simulations, for simplicity, polymers are assumed to be fully charged and aspects of their single chain behavior (aspects of compaction) and multiple chain behavior (aspects of aggregation) in the presence of counterions have been explored. As salt occurs universally in physiological environment, there is an increasing need to understand the role played by solvent, specific nature of salt or counterions in the solution and the structural conformational entropy of biological charged polymers themselves ${ }^{42-47}$.

The presence of salt in the process of protein aggregation has attracted wide attention because of its profound effect on protein aggregation, protein stability and protein solubility ${ }^{48-54}$. Previous studies have 
explained the effect of salt on protein aggregation in terms of various models such as the Debye-Huckel screening of charges, effects on the protein-water-ion interactions (Hofmeister series) and specific ion binding (electroselectivity series). Some of the parameters such as $\mathrm{pH}$, temperature, presence of co-solutes etc., have been shown to influence the self-assembly of proteins in diverse ways as reported in several studies. Recently the experimental kinetic studies of salt-induced aggregation on a model antibody using different types of salts at various $\mathrm{pH}$ values have shown that salt concentration promotes aggregation through the formation of protein intermediates characterized by partially ordered secondary structures and is strongly dependent on ion's specificity and $\mathrm{pH}$ conditions ${ }^{54}$. In the experimental studies on the yeast prion protein Sup35, salts were found to follow Hofmeister series in modulating the aggregation propensity ${ }^{53}$ whereas specific anion binding was shown to play a crucial role in the aggregation process of mouse prion protein and b2-microglobulin ${ }^{48,55}$. In the case of Alzheimer's A $\beta(1-40)$ peptide both of the Hofmeister effect and anion binding effect play an important role on aggregation propensity and the aggregation was found to be progressively more favourable as the salt concentration was increased from $50 \mathrm{mM}$ to $500 \mathrm{mM}^{52}$. The heat induced aggregation kinetics of Human carbonic anhydrase II was also found to be highly sensitive to salt concentration ${ }^{49}$. There is also a report where different Hofmeister sequences are effective in the different concentration regimes of the salts ${ }^{56}$.

In this work, our primary objective is to probe the effect of different salts at moderately high concentration, and their valency on the aggregation of an explicitly solvated charged polypeptide system. In the present study, the aggregation dynamics of a hexapeptide ( 57,58 VEALYL, referred henceforth as IB12), a segment from the $\mathrm{B}$ chain of the fibril-forming protein insulin, was explored using atomistic molecular dynamics. This particular short peptide was selected in our studies of probing atomistically aggregation process for computational feasibility and also, it has been investigated earlier as a model system for protein aggregation studies ${ }^{59,60}$. Insulin, a small hormone consisting of $\alpha$-helix and cross$\beta$ structure has become a model peptide for studying fibril formation because of it's simple structure. Under certain conditions (elevated temperatures, low $\mathrm{pH}$, hydrophobic interface, ionic strength, and mechanical agitation $)^{61,62}$ it undergoes denaturation and further aggregates in fibril-like structures via fibrillation. Previous simulations ${ }^{59,60}$ on IB12 peptide using GROMOS force-field parameters reported an abundance of $\beta$-sheet rich aggregate formation from unstructured monomers and so, those studies are primarily focused on the initial aggregation events of fiber formation (natural or amyloid). However, from the oligomerization of IB12 peptide sequences, it is becoming increasingly apparent that it may be the prefibrillar (often amorphous and granular) aggregates that produce cytotoxic effects in vivo $^{8-11}$. These require detailed atomistic simulations with correctly parameterised forcefields to capture the essential aspects of the proteins, water and salts added and can be very expensive computationally to explore the full aggregation pathways. It is more feasible, at atomistic resolution, to explore the initial pathways of aggregation, from which valuable insights into the early stage kinetics of aggregation can also be gained ${ }^{59,60,63,64}$. Here we focus on the detailed understanding of the pathways of formation (and degradation) of amorphous aggregates, which are likely to be fundamentally different from the mechanisms of fibril growth. Several chains of IB12 (net charge=-1) peptide system were dispersed into three types salts namely $\mathrm{NaCl}, \mathrm{KCl}$, and $\mathrm{MgCl}_{2}$ solution at $1.0 \mathrm{M}$ concentration and the role of salt condition in accelerating and/or retarding the amorphous aggregation is discussed here. Attempts have been made to identify specific molecular interactions that direct the association of IB12 peptide monomers leading to the formation of stable aggregates in the various salt-solutions at molar level concentration. Our results provide mechanistic insight into the underlying physical processes of amorphous aggregation and explain the dependence of aggregation properties on the nature of salt by the detailed analyses involving Molecular Mechanics Generlised Born Surface Area (MM/GBSA) model, Charge renormalizatin of aggregates by salt-ions and Markov State Model (MSM). The MM/GBSA model has been used successfully in several areas of protein biophysics such as binding affinities proteinligand, protein-protein and multi-component protein interaction $^{65,66}$. MSMs $^{67-71}$ are generally used in kinetic studies of folding and unfolding events of nucleic acids and proteins ${ }^{72}$. The charge renormalization of highly charged macroions has been observed when the macrions undergo a condensation in solutions in the presence of multivalent counterions ${ }^{73-75}$. Here the combination of MM/GBSA, MSM and Charge renormalization method will be important to protein aggregation studies as well.

The rest of the article arranged as follows: in section II we discuss the procedure to set up the systems, simulation protocols employed and methods used for our calculation. The results obtained are presented in section III, followed by discussions on the important findings of this study and the conclusions in section IV.

\section{METHODS}

Aggregation of peptides IB12(Val-Glu-Ala-Leu-TyrLeu $)^{59,60}$ is studied using all-atom classical molecular dynamics methods. Several IB12-solvated systems were prepared with $1.0 \mathrm{M}$ concentration of salts (both monovalent and divalent) and TIP $3 \mathrm{P}^{76}$ water model. TIP4P$\mathrm{Ew}^{77}$ water model was also used to analyse if the aggregation behavior depends strongly on the water model used. 
All results in the paper are for TIP3P water model and the results from TIP4P-Ew are available in Sec. I of supplementary material. For ions, standard CHARMM parameters used extensively in biomolecular systems with TIP3P water model ${ }^{78}$ and similar parameters specific to TIP4P-Ew water model were used ${ }^{79,80}$. Each IB12 peptide carries a total charge of -1 due to the presence of Glu amino acid.

\section{A. System setup}

The starting configuration of IB12 peptide structure was generated using molefacture plugin in $\mathrm{VMD}^{81}$ and the single IB12 peptide was solvated in TIP3P water box $(\approx 38.5 \AA \times 38.5 \AA \times 38.5 \AA)$ and neutralized by adding a single $\mathrm{Na}^{+}$ion in the water box. Initially the system was relaxed using conjugate-gradient energy minimization algorithm by 1000 steps and equilibrated for $5 \mathrm{~ns}$ under isothermal-isobaric (NPT) ensemble condition at pressure of $1 \mathrm{~atm}$ with a timestep of $2 \mathrm{fs}$. Next, 64 replicas of equally spaced equilibrated IB12 peptides were solvated in a cubic box of size $108 \AA^{3}$. Multiple systems were derived from this primary one by ionizing with $1.0 \mathrm{M}$ concentration of $\mathrm{NaCl}, \mathrm{KCl}$ and $\mathrm{MgCl}_{2}$ salts using autoionize plugin of VMD. A separate control system was also prepared with addition of ions only to neutralize the total peptide charge. The systems were then equilibrated with 1fs timestep for $5 \mathrm{~ns}$ under NPT ensemble by fixing the center of mass locations of the Ala residue in each peptides so that initial aggregation is prevented, allowing water and ions to equilibrate around the peptides. The final conformation for each case, at the end of this 5ns of equilibration run was used as initial system configuration for the subsequent production (NVT) runs. Two independent 100ns long simulations were performed for each case. All the system and simulation details are listed in Table I.

TABLE I. System details and summary of performed simulations for IB12 peptides in TIP3P water model.

\begin{tabular}{cccccc}
\hline salt & salt conc. & $\begin{array}{c}\text { No of } \\
\text { cations }\end{array}$ & $\begin{array}{c}\text { No of } \\
\text { anions } \\
\left(\mathrm{Cl}^{-}\right)\end{array}$ & & \\
time(ns/run) & no. of sim \\
\hline $\mathrm{NaCl}$ & $1.0 \mathrm{M}$ & 675 & 739 & $100 \mathrm{~ns}$ & 2 \\
$\mathrm{MgCl}_{2}$ & $1.0 \mathrm{M}$ & 707 & 1350 & $100 \mathrm{~ns}$ & 2 \\
$\mathrm{KCl}$ & $1.0 \mathrm{M}$ & 675 & 675 & $100 \mathrm{~ns}$ & 2 \\
\hline
\end{tabular}

\section{B. Simulation protocols}

All MD simulations were performed using simulation package NAMD $2.9^{82}$ using CHARMM $36^{83}$ parameters for peptides. The MD simulations were performed at a temperature of $298 \mathrm{~K}$ in NVT ensemble. For NPT runs, a pressure of $1 \mathrm{~atm}$ was maintained using Nosé-HooverLangevin piston ${ }^{84}$ with a decay period of $100 \mathrm{fs}$ and a damping time of $50 \mathrm{fs}$. The non-bonded cut-off distance was set to $12 \AA$ with a switching distance between $10 \AA$ and $12 \AA$. The long-range electrostatic interactions were treated by Particle mesh Ewald (PME) ${ }^{85}$ method and the data visualization and analyses were done using the software $\mathrm{VMD}^{81}$ and in house data analysis scripts.

\section{Markov state model analysis}

To investigate the time-evolution of aggregate size distribution Markov State Model (MSM) approach was used for analyzing the MD trajectories. MSM provides a convenient way to model kinetic network for conformational transitions ${ }^{67-71}$. In MSM formalism, the dynamics of the system is described in terms of state-to-state transitions and the time-evolution of the system is governed by the discrete-time master equation:

$$
\pi(t=n \tau)=\pi(t=0) P(n \tau)=\pi(t=0)[P(\tau)]^{n}
$$

where $\pi$ is the row vector of probabilities of occupying any of the Markov states at time $t$ and $P(\tau)$ is the transition probability matrix, whose entries, $P_{i j}$, provide the probability of the system to be found in state $j$ at time $t+\tau$ given that it was in state $i$ at time $t . \tau$ is the time interval of obersevation, called the lag time.

Here, MSM describes the dynamics among the aggregate states using a transition matrix, $T$. The components of $T, T_{i j}$ were computed by counting the total number peptides transitioning from the aggregate state of $i$-mer $\left(S_{i, t}\right)$ to aggregate state of $j$-mer $\left(S_{j, t+1}\right)$ in next time step. Here $S_{i, t}$ and $S_{j, t+1}$ represent an aggregate size state of $i$-mer and $j$-mer at time $t$ and $t+1$ respectively. A $64 \times 64$ transition probability matrix, $P$ was constructed by normalizing the transition matrix elements with the sum of elements contained in the corresponding row. Mathametically we can express this term as follows;

$$
P\left(S_{i}, S_{j}\right)=\frac{\sum_{t=0} \operatorname{Count}\left(S_{i, t} \rightarrow S_{j, t+1}\right)}{\sum_{t=0} \sum_{j=1}^{64} \operatorname{Count}\left(S_{i, t} \rightarrow S_{j, t+1}\right)}
$$

Thus, the fraction of aggregate size $(N$-mer) in the trajectory after $n$ propgation steps can be obtained by solving the master equation as row vector $\pi(n)=\pi(0) P^{n}$. Here $\pi(0)$ is the row vector containing the starting fractional populations as $\pi\{1,2, \ldots ., 64 ; \mathrm{t}=0\}=\{1,0, \ldots, 0\}$. 


\section{RESULTS}

\section{A. Aggregate morphology}

In this section the dependence of the size of the peptide aggregates and their morphology on the concentration and valency of the salts are described. At any given instant, two peptides are defined to be part of the same aggregate if the distance between two non- $\mathrm{H}$ atoms in the respective peptides is less than $3 \AA$. The representative snapshots of aggregate states for all three salts under $1.0 \mathrm{M}$ concentration and the control simulaton at the end of 100ns, are shown in Figure 1. The individual peptides are colored based on the size of the aggregate they belong to. From the Figure 1a, it can be seen that for systems with $\mathrm{NaCl}$ salt, the number of aggregates is smallest, with one single aggregate being the predominant aggregate morphology. This is in contrast to the control system (Figure 1d), where the number of aggregates is larger and the average aggregate size is much smaller. The typical aggregate morphologies in other salt systems is also shown in the Figure 1. In the case of $\mathrm{KCl}$ (Figure 1c), also a monovalent salt, the aggregation behavior is very different from that of $\mathrm{NaCl}$ case, strongly underscoring the influence of type of cation on such aggregation processes and not just the valency of the cation. In the case of divalent salt $\mathrm{MgCl}_{2}$ (Figure 1b), it can be seen that the though aggregate size is larger than $\mathrm{KCl}$ and control systems, the system does not display the near phase separated aggregate morphology seen in $\mathrm{NaCl}$ systems. These visual results suggest that the aggregate behavior of peptides in salt-water systems are function of not only the valency of the cation, but are strongly dependent on the cation type as well.

To quantify the visual results in Figure 1, the morphology of the aggregates is quantified in terms of normalized aggregate size distribution, over the last 10ns, which for all the systems is shown in Figure 2. In Figure 2 the aggregate size $(S)$ is the total number of peptides forming a given aggregate. Apart from a few stray single peptides, the distribution of the aggregate size for $1.0 \mathrm{M} \mathrm{NaCl}$ salt solution, peaks around $\sim 58$ at the end of $100 \mathrm{~ns}$ long simulation suggesting that for this case, almost all of the peptides are in an isolated aggregate. The distribution of aggregate sizes for $\mathrm{KCl}$ and $\mathrm{MgCl}_{2}$ suggests that the propensity in these systems is not towards one single large aggregate, but a distribution of aggregate sizes corresponding to the well known finite bundle model suggested in literature ${ }^{86-89}$. In finite bundle model, the finite-sized bundle-like aggregates of the charged macromolecular system in the presence of multivalent salt are suggested to be the thermodynamic equilibrium states. However, in the control system where no extra salt is added, the distribution of aggregate sizes is restricted to smaller sizes, clearly showing that the effect of addition of salt, in general, is to promote (a) IB12+1.0M NaCl+Water

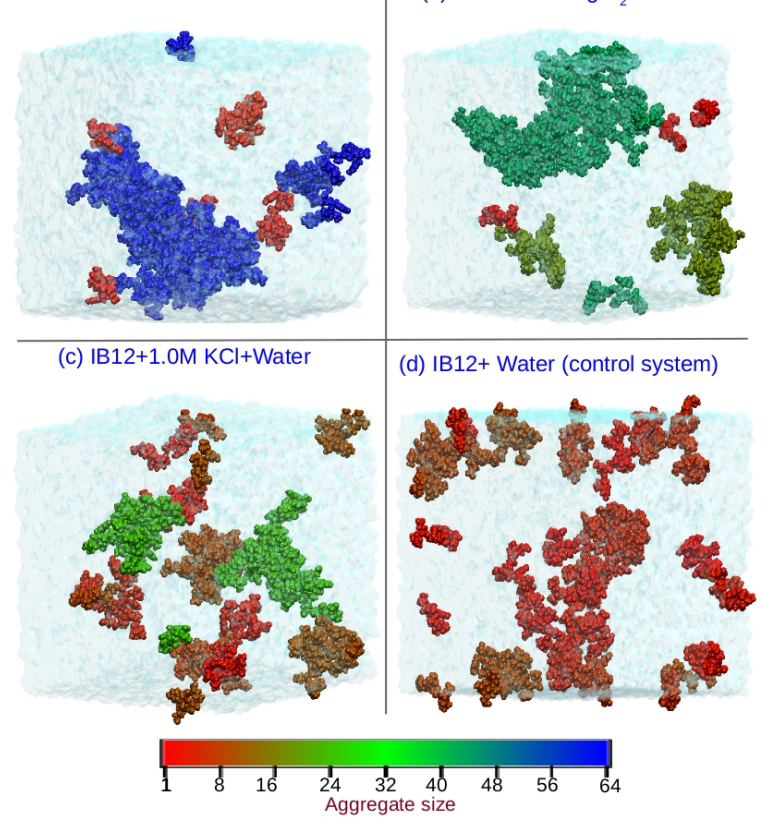

FIG. 1. MD snapshots (in VDW representation) of the endproduct in one of the 100ns long IB12 peptide simulations for $\mathrm{NaCl}(\mathrm{a}), \mathrm{MgCl}_{2}$ (b), $\mathrm{KCl}(\mathrm{c})$ salt and control system(d) respectively. The coloring of the peptide is based on the aggregate size they belong to. The salt-ions are not shown for clarity.

the aggregation though differences may exist in the morphologies of the same depending on the valency and type of the cation. The increase of aggregate sizes in the presence of $\mathrm{NaCl}$ salt, compared to control system seen in our simulations agrees with similar increase in aggregate sizes seen in experiments ${ }^{90}$ on chitosan based charged polymers. These experiments also suggested that the aggregation morphologies in presence of divalent salts is different from that of monovalent salts.

One of the measures of aggregate size is radius of gyration $\left(R_{\mathrm{g}}\right)$ and particularly its variation with time. In Figure $3, R_{\mathrm{g}}$ of the largest aggregate is plotted as a function of time in the last 25ns of simulation to understand the differences in the size distribution of the aggregates between $\mathrm{NaCl}$ and $\mathrm{KCl}$ systems though in both systems the cation is monovalent. The data in Figure 3 shows that even though the aggregate size for $\mathrm{KCl}$ solution is much smaller than that of $\mathrm{NaCl}$, the $R_{\mathrm{g}}$ of the largest cluster for $\mathrm{KCl}$ (33 peptides) is similar or larger than that of $\mathrm{NaCl}$ case (58 peptides) indicating the possibility of more compact structure formation of the peptides in the presence of $\mathrm{NaCl}$ salt. This can also be visually seen from Figure 1c, where the aggregates, in the presence of $\mathrm{KCl}$, are more loosely packed.

To quantify the compactness of the aggregates, we 


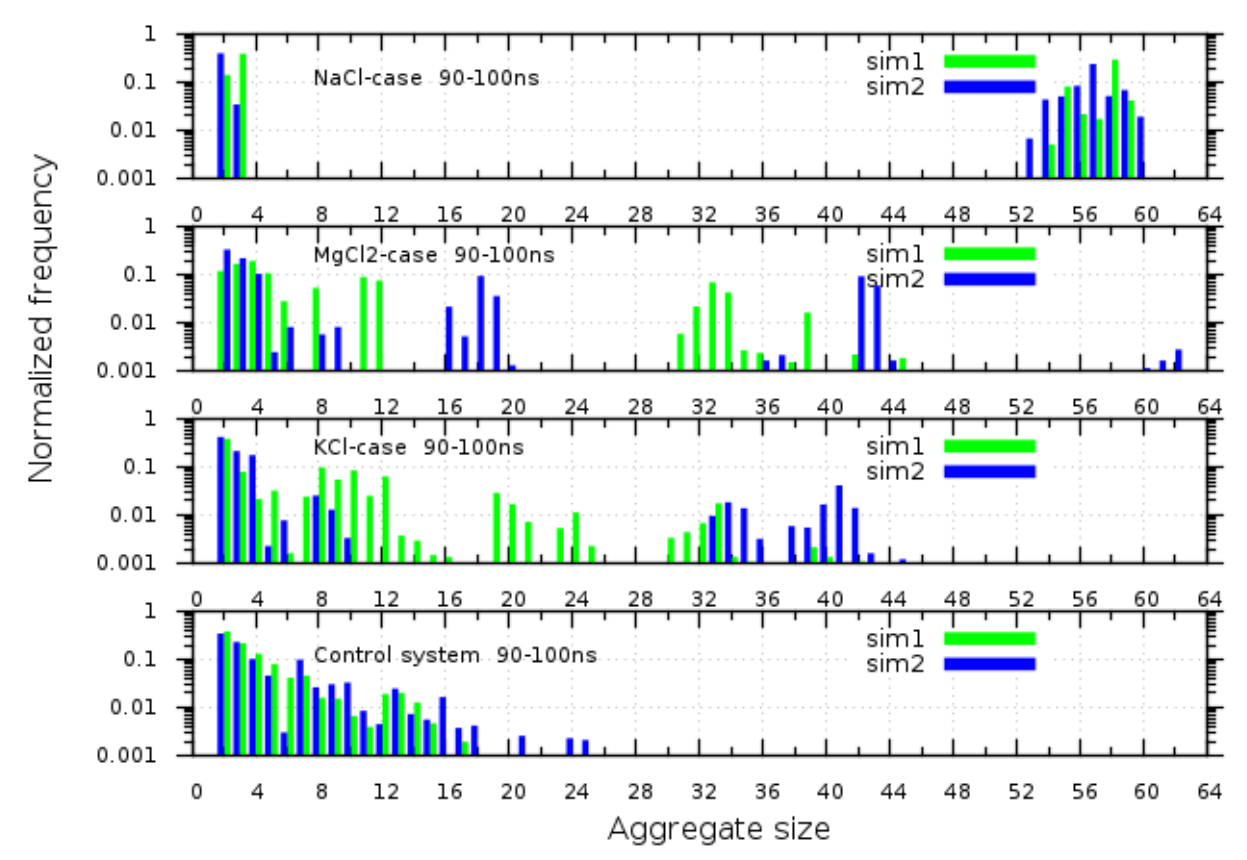

FIG. 2. Normalized aggregate size distribution for IB12 system with $\mathrm{NaCl}, \mathrm{MgCl}_{2}, \mathrm{KCl}$ salt-solutions and control system computed from last 10ns simulation data for each trajectories.

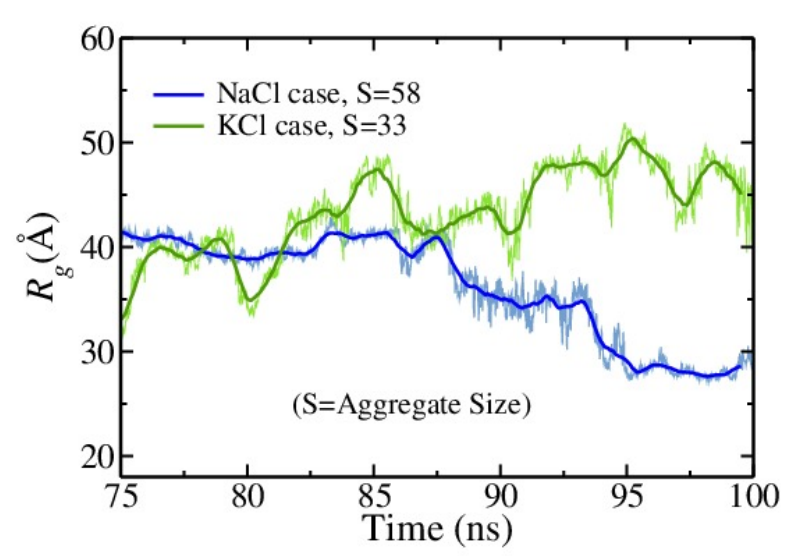

FIG. 3. Time evolution of radius of gyration $\left(R_{\mathrm{g}}\right)$ of large peptide aggregate for IB12-NaCl and IB12-KCl systems in the last $25 \mathrm{~ns}$ of simulation. $R_{\mathrm{g}}$ values are calculated for the peptide chains belong to aggregate of size 58 and 33 for $\mathrm{NaCl}$ salt and $\mathrm{KCl}$ salt solutions respectively.

compute the pair distribution function $\mathrm{g}(\mathrm{r})$ for hydrophobic residues of the peptides as shown in Figure 4. The peptide composition, as seen in Methods, shows that there is a single charged amino acid GLU and has three hydrophobic residues (VAL, ALA, LEU). From the $\mathrm{g}(\mathrm{r})$ data, it can be seen that the location

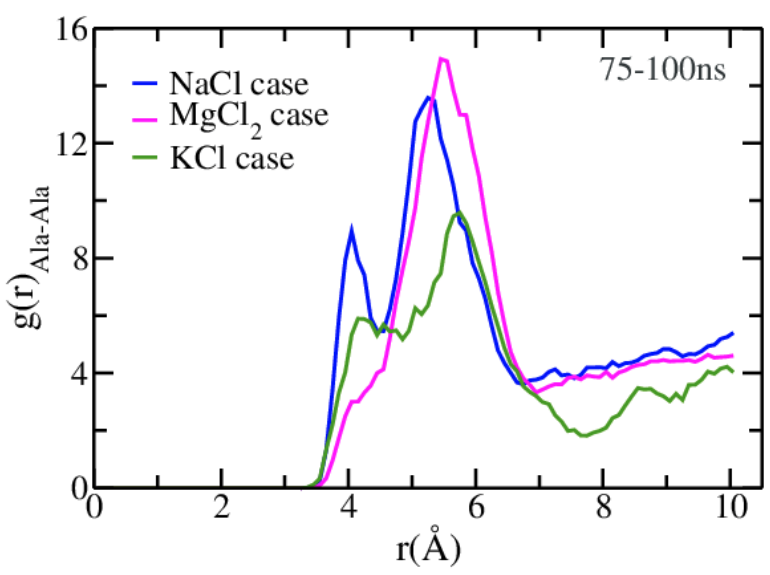

FIG. 4. The pair correlation function $(\mathrm{g}(\mathrm{r}))$ of the hydrophobic residues Ala of the IB12 peptide for three types of salt solutions.

and peak heights for ALA-ALA distribution is at a shorter distance for $\mathrm{NaCl}$ than for $\mathrm{KCl}$. This can be attributed due to different sizes of the cations. $\mathrm{NaCl}$ promotes the aggregation of hydrophobic residues much more compared to the $\mathrm{KCl}$ and the most probable closest distance between hydrophobic residues is much larger for the case of divalent salt $\mathrm{MgCl}_{2}$. Some of the earlier simulations on small hydrophobic solutes 
and hydrophobic polymers in salt solutions have shown strong stabilization of these hydrophobic interactions in the presence of $\mathrm{NaCl}^{91}$. These observations strongly suggest that valency and nature of salt play a crucial role on the morphology of the aggregates and the aggregate sizes. A possible understanding of dependence of critical size of aggregates and the valency of the ions is discussed in latter sections.

A visual inspection of the IB12 simulation trajectories suggested that there was a conversion from random coil to extended $\beta$-sheet conformation as the simulation time progresses which is generally in line with earlier literature ${ }^{59}$. In a couple of cases, there were signatures of $\beta$-sheet formation between two peptides but they were not so substantial. Representative snapshots of secondary structures from one of the IB12 simulations are shown in Figure 5 for $\mathrm{NaCl}$ and $\mathrm{MgCl}_{2}$ salt solutions. For both cases very few monomers ( $\sim 4-8$ strands) were found to form parallel, anti-parallel or disordered betasheet conformation. In any case, $\beta$-sheet formations are expected to take place at considerably longer timescales. However, in the present study we are interested in the early stage of salt-induced aggregation events of charged peptides rather than conformational dynamics.

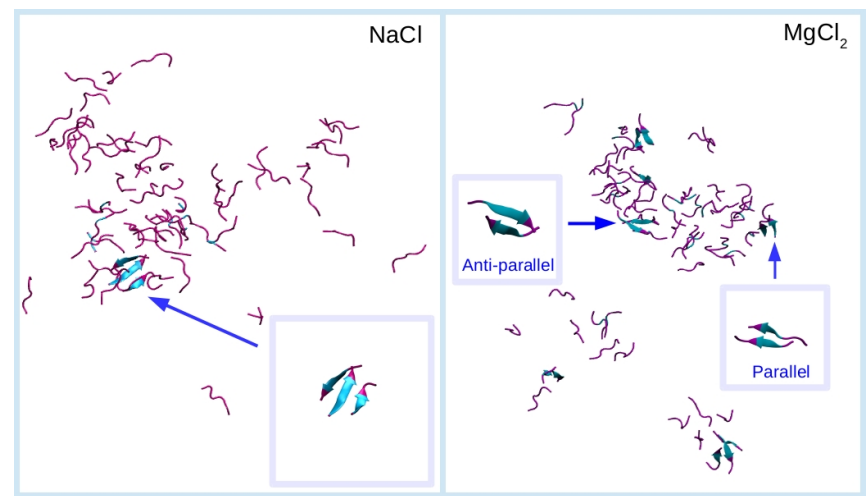

FIG. 5. Snapshots of secondary structures observed at the end of 100ns long trajectory of IB12 in $\mathrm{NaCl}$ and $\mathrm{MgCl}_{2}$ salt solution. Two set of representative peptide aggregates endstructure (at 100ns) are depicted in cartoon representation, showing ordered or disordered $\beta$-sheet assemblies. The colors encode $\beta$-sheet (cyan) and random coil (magenta) secondary structure elements.

To understand the effect of salt on the dynamics of peptide aggregation at molecular level, several comparative analyses between different kind of IB12-salts and control system have been done. In next section we describe the dynamic evolution of peptide aggregates.

\section{B. Dynamic evolution of peptide aggregates}

Figure 6(a) presents a comparison between the evolution of the total the number of aggregates in the four systems. In Figure 6(a), $N(t)$ is the the number of
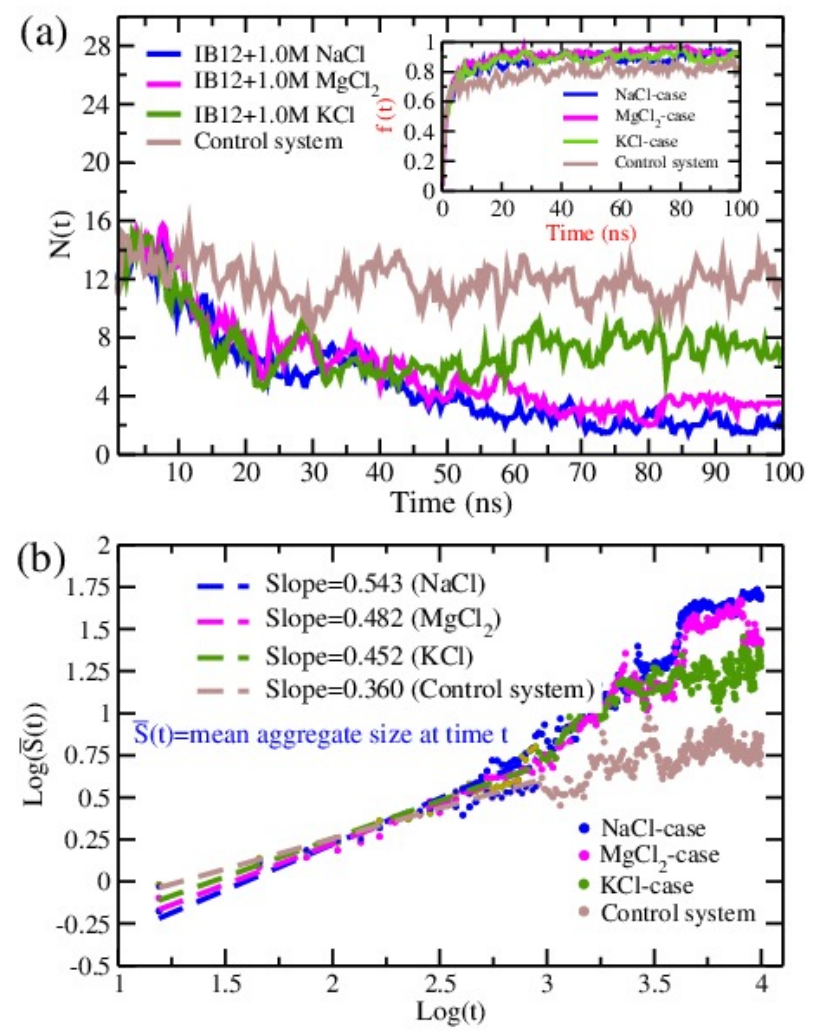

FIG. 6. (a) Dynamic evolution of the total number of aggregates $N(t)$ excluding single peptide state i.e. aggregate size $(S)>=2$. The blockavearge of data were obtained from each time slice of $300 \mathrm{ps}$. Inset shows the time evolution of the fraction of total no. of peptide chains forming aggregates. (b) Dynamic evolution of the mean aggregate size $\bar{S}(t)$ in the 64-peptide system. The dashed line, representing fit of $\bar{S}(\mathrm{t})$ at timescale of $0-10 \mathrm{~ns}$. The slope of the straight lines are about $0.543,0.482$ and 0.452 for $\mathrm{NaCl}, \mathrm{MgCl}_{2}$ and it is $\sim 0.360$ for control system respectively.

aggregates at time $t$ with aggregate size, $S>=2$. In complete phase separated state, $N(t)=1$ i.e. one single aggregate containing 64 peptide chains and in complete deaggregated state, it is 0 . For finite bundle ${ }^{86-89} N(t)$ lies somewhere in between 1-32 for 64-mer peptide system. In plot Figure 6(a) we see that the peptides started to aggregate from very early stage of simulation for all four cases. The plot suggests that assembly towards the final oligomeric state is faster in the presence of salt as compared to control system. It is northworthy that aggregation is relativey faster for $\mathrm{NaCl}$ case than $\mathrm{KCl}$ salt at long-timescale. A slight difference in aggregation rate was observed between $\mathrm{NaCl}$ and $\mathrm{MgCl}_{2}$ salt beyond $60 \mathrm{~ns}$. The deaggregation events of IB12 multi-peptide 
system in $\mathrm{MgCl}_{2}$ at timescale between $60-80 \mathrm{~ns}$ is accounting for this difference in aggregation rate. The time evolution of the fraction of peptide chain taking part in aggregate formation are shown in Figure 6(a) inset. Our result shows that almost $90 \%$ of peptide chains (out of 64) formed aggregate in the presence of salt whereas the fraction is around $80 \%$ for control system, which also has a propensity of forming substantially smaller aggregates.

In Figure 6(a) we observe that initially the growth of aggregates size proceeds rapidly for all cases and beyond $75 \mathrm{~ns}$ timescale the aggregate phase were emerged to be more stable. Large stable aggregates were formed at timescale of $75 \mathrm{~ns}$. The average number of aggregates formed at time scale of 75-100ns are 2.11, 3.34, 7.54, 11.738 for $\mathrm{NaCl}, \mathrm{MgCl}_{2}, \mathrm{KCl}$ and control system repectively.

For $1.0 \mathrm{M} \mathrm{MgCl}_{2}$ salt solution, at the timescale of $75 \mathrm{~ns}$ a large cluster with aggregate size $>60$ was found to be formed in one of our 100ns trajectories but as the time progresses deaggregation took place and the larger cluster collapsed into two smaller clusters of sizes $\sim 33$ and $\sim 18$. The aggregate formation of the peptide system was found to be more stable in $\mathrm{NaCl}$ salt solution compared to $\mathrm{MgCl}_{2}$. We can define the mean aggregate size $(\bar{S}(t))$ at time $t$ by the standard formula used in aggregation of clusters (Refs. ${ }^{92,93}$ )

$$
\bar{S}(t)=\frac{\sum_{S} S^{2} n_{s}(t)}{\sum_{S} S n_{S}(t)}
$$

where $n_{S}$ is the number of aggregates (excluding single peptides) that contain $S$ peptide chains. Figure 6(b) shows $\bar{S}(t)$ as a function of time. At short timescale, $\bar{S}(t)$ is found to grow with time as a power law but, the dynamic scaling for aggregation of clusters is not followed at long timescales. The lines, representing linear fit of $\bar{S}(t)$ at short time, have a slope of about $0.543,0.482$ and 0.452 for $\mathrm{NaCl}, \mathrm{MgCl}_{2}$ and it is $\sim 0.360$ for control system.

To monitor the deaggregation events in salt solution we put our focus on the aggregate state $\left(S_{i, t}\right)$ associated with each peptide chain at each time step $t$ which are represented by Figure 7 . Figure 7 depicts the time series of aggergate size states associated with each peptide for $\mathrm{NaCl}$ and $\mathrm{MgCl}_{2}$ salt along $\mathrm{y}$ axis, where the $\mathrm{x}$ axis labels the peptide segment running from $\mathrm{S} 1$ to S64 and the colorscale being a mark of aggregate size state $\left(S_{i}\right)$ to which a particular peptide belongs. Here $S_{i}$ represents an aggregate size state of $i$-mer. In matrix representation of following figures, the decrease in intensity from blue to green in any column implies the occurance of disassociation event. At the early stage of aggregation the peptide molucules were found to be associating and disassociating reversibly, eventually
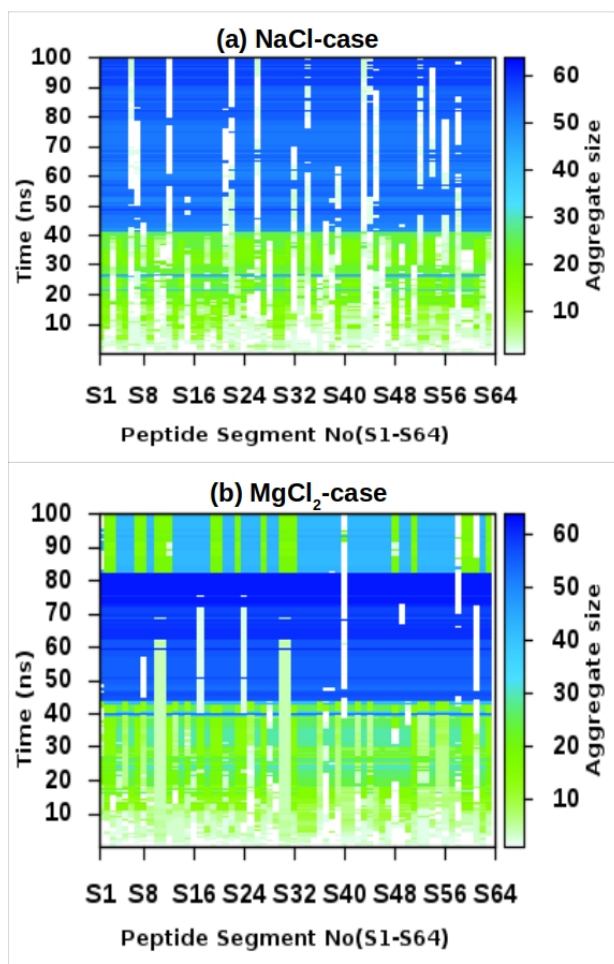

FIG. 7. Matrix representation of the time evolution of aggregate size state $\left(S_{i}\right)$ of each peptide monomer for $\mathrm{NaCl}(\mathrm{a})$ and $\mathrm{MgCl}_{2}$ (b) salt-solution. The $\mathrm{x}$-axis denotes the labeling of peptide segments running from S1-S64 and the color scale encodes the aggregate size state $S_{i}$.

large aggregates were formed between 75-80ns. Beyond 75ns timescales, deaggregation events have been observed predominantly for $\mathrm{MgCl}_{2}$ salt compared to two other salts. This result suggests us that the presence of divalent atom may have destabilising influence on the association events of peptide molecules. So an understading of the interaction between the protein, ion and water molecules can allow us to get better insight into the mechanism of aggregation process in the presence of salts, those are follwed in the succeeding sections.

\section{Interaction between water, salt-ions and peptides}

To assess the interaction among the peptide, salt and water molecules we computed the radial distribution function, $\mathrm{g}(\mathrm{r})$ for various cases. The radial pair distribution function (pair correlation function) provides us valuable insights into the distribution of atoms (molecules) around another atom (molecules) of same kind or different kind. First we calculate the distribution of salt-cations around Glutamic acid (Glu) of peptide molecule from 75-100ns simulation data as shown in Figure 8 . 


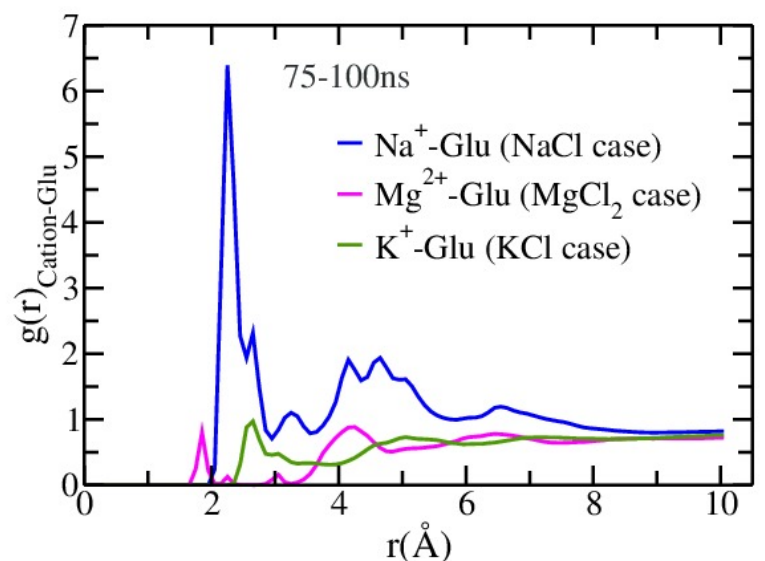

FIG. 8. Radial pair distribution function $\left(\mathrm{g}(\mathrm{r})_{\text {Cation-Glu }}\right)$ of cations of salt around the negatively charged residue Glu of the peptide in salt-water solution obtained from last $25 \mathrm{~ns} \mathrm{MD}$ data.

The $\mathrm{g}(\mathrm{r})$ results clearly show that $\mathrm{Na}^{+}$ions interact most strongly with Glu residues compared to both $\mathrm{K}^{+}$ and $\mathrm{Mg}^{2+}$ cations. This also indicates that the total number of cations within $3 \AA$ of residue $\mathrm{Glu}$ in $\mathrm{NaCl}$ case is much higher compared to other two salts and also that more $\mathrm{Na}^{+}$cations are interacting with Glu in comparison with $\mathrm{K}^{+}$and $\mathrm{Mg}^{2+}$ ions. For $\mathrm{MgCl}_{2}$ salt though a peak appears at smaller distance compared to $\mathrm{NaCl}$, the effective distance between a typical $\mathrm{Mg}^{2+}$ cation and Glu is much larger and is greater that $4 \AA$ compared to the $2 \AA$ most probable distance between $\mathrm{Na}^{+}$and Glu residues. The order of the strength of ion-pair interaction in aqueous solution of three salts was found as $\mathrm{NaCl}<\mathrm{KCl}<\mathrm{MgCl}_{2}$ from the calculation of $\mathrm{g}(\mathrm{r})$ of anions around cations (see Sec. II of the supplementary material). The interaction of salt-ions with water also plays a crucial role in solubility of a salt in water as the solubility is the result of the balance between two competing interactions: the ion-pair interaction of the salt and the ion-water interaction. From the $\mathrm{g}(\mathrm{r})$ analysis of water-cation interaction we observed a welldefined strong hydration shell around $\mathrm{Mg}^{2+}$ ions with an additional outer hydration layer whereas for monovalent salts, $\mathrm{Na}^{+}$and $\mathrm{K}^{+}$ions exhibit weaker interaction with water (see Sec. II of supplementary material). However, any effects of high salt concentration of salts on water's bulk structure were not studied in our simulations. We noticed that within the hydration shell around $\mathrm{Mg}^{2+}$ the oxyzen atoms of hydrating water molecules pointing towards the $\mathrm{Mg}^{+}$ion make relatively stronger interaction due to divalency and negative hydration entropies of smaller $\mathrm{Mg}^{2+}$ ions compared to realatively larger monovalent ions (the snapshots of condensed anions and water molecules around a single cation for three types of salt-solutions are shown in Sec. II of sup- plementary material). These results are consistent with the Hofmeister series ranking chaotropes (weakly hydrated) to kosmotropes (strongly hydrated) for cations as $\mathrm{NH}_{4}^{+}>\mathrm{Cs}^{+}>\mathrm{Rb}^{+}>\mathrm{K}^{+}>\mathrm{Na}^{+}>\mathrm{Li}^{+}>\mathrm{Ca}^{2+}>\mathrm{Mg}^{2+94-97}$. It is conceivable that in the case of $\mathrm{MgCl}_{2}$ salt, the water molecules within the first hydration shell of $\mathrm{Mg}^{2+}$ ion are so tightly bound that $\mathrm{Mg}^{2+}$ ions are less available for peptide atoms.

Also, other factors like the charge, size, polarizability, temperature, pressure and concentration of the ions contribute to the the solvent dynamics, mobility and solulibility of salts. The vdW radii of three metal ions studied here are $2.27 \AA, 1.73 \AA, 2.75 \AA$ for $\mathrm{Na}, \mathrm{Mg}$ and $\mathrm{K}$ atom respectively ${ }^{98}$. Now, we define the ions/atoms of type A which interacts with another atom/molecule of type $\mathrm{B}$ within $3 \AA$ as condensed ions/atoms $\mathrm{A}$ around atom/molecule B. For condensed metal ions around the negatively charged Glu amino acid, the distances between the metal-ion and Glu residue averaged over last 10ns simulation data, 2.42 and 2.57 for $\mathrm{NaCl}$ and $\mathrm{MgCl}_{2}$ respectively confirm the effective larger size of $\mathrm{K}^{+}$than $\mathrm{Na}^{+}$ion. So not only the extent of ion-pair interaction also the smaller size of the $\mathrm{Na}$ atom than $\mathrm{K}$ may cause a pronounced interaction of $\mathrm{Na}^{+}$ion with amino acid Glu of peptide over $\mathrm{K}^{+}$ion as small cations are likely to bind on the surface of charged polypeptide. A comparison of the ratio $\left(t_{c}\right)$ of condensed cations

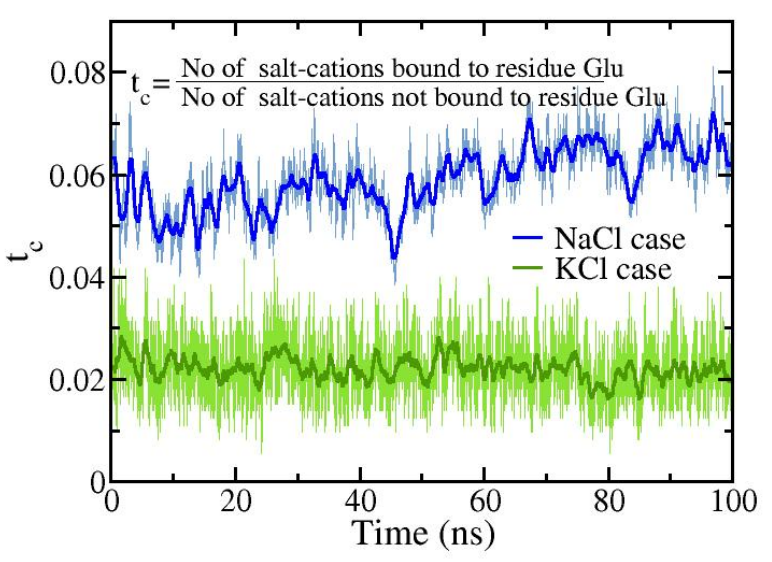

FIG. 9. The ratio $\left(t_{c}\right)$ of the no. of salt-cations $\left(\mathrm{Na}^{+}\right.$and $\mathrm{K}^{+}$) bound to Glu and no. of salt-cations not bound to Glu in simulation as a function of time.

around Glu and metal ions unbound to residue Glu as a function of time is shown in Figure 9. In Figure 9, the increase in the interaction of $\mathrm{Na}^{+}$ions with Glu with time is an indicator of the influence of free $\mathrm{Na}^{+}$ions within bulk-water on the growth of aggregates whereby the interaction is constant on average in $\mathrm{KCl}$ case. The average number of condensed cations and anions for various cases are listed in Table II. Table II shows a 
weaker interaction of $\mathrm{Cl}^{-}$ions with protein heavy-atoms.

TABLE II. Number of condensed ions averaged over last 10ns MD data.

\begin{tabular}{llll}
\hline Salt & $\begin{array}{c}\text { Mean aggre- } \\
\text { gate size } \\
(\bar{S})\end{array}$ & Cation-Glu & \\
& \multicolumn{1}{c}{ CLA-peptide } & \\
$\mathrm{NaCl}$ & 51.167 & 43.9105 & 0.78 \\
\hline $\mathrm{MgCl}_{2}$ & 27.62 & 4 & 1.812 \\
\hline $\mathrm{KCl}$ & 19.18 & 15.68 & 0.638 \\
\hline $\begin{array}{c}\text { a Average number of condensed salt-cations around } \\
\text { Glu residue. } \\
\text { bverage number of condensed } \\
\text { heavy atoms of peptide. }\end{array}$
\end{tabular}

Further the primary free-energy contributions of the peptide atoms that lead the polypeptide system toward forming the aggregates have been discussed in next section.

\section{Electrostatic, vdW interaction energy and solvation free energy of aggregates}

Aggregation of peptides is usually considered to be a result of absence of repulsive electrostatic forces, the deceasing effect of solvation free energy of peptides and short-range of vdW interaction between peptides ${ }^{99}$. The decreased effects of solvation are attributed to the absence of H-bonds with water molecules and the formation of interpeptide H-bonds. Here, we estimate the binding energy between the peptides using Molecular Mechanics Generlised Born Surface Area (MM/GBSA) ${ }^{100}$ method. According to MM/GBSA the binding free energy $\left(\Delta G_{b i n d}\right)$ of the peptide molecules to form aggregates can be estimated from the following sum,

$$
\begin{aligned}
\Delta G_{\text {bind }}=< & \Delta G_{\text {elec }}>+<\Delta G_{v d w}> \\
& +<\Delta G_{\text {solv }}>-T \Delta S
\end{aligned}
$$

where the first two terms are standard molecular mechanics (MM) contributions originating from electrostatic and van der Waals interactions of aggregates, $\Delta G_{s o l v}$ is the total solvation free energy obtained from the polar $\left(\Delta G_{\text {polar }, \text { elec }}\right)$ and non-polar $\left(\Delta G_{n p}\right)$ contributions, and $T \Delta S$ corresponds to the entropic contribution. Here, non-boned interaction energy (vdW energy and electrostatic energy) of peptide molecules and electrostatic solvation free energy ( $\left.\Delta G_{\text {polar, elec }}\right)$ have been computed by 'namdenergy' plugin of VMD. The nonpolar solvation energy $\left(\Delta G_{n p}\right)$ can be estimated from a linear relation to the solvent accessible surface area (SASA) ${ }^{101,102}$ given by,

$$
\Delta G_{n p}=\gamma \times S A S A+a
$$

where $\gamma$ and a are constants. In our analysis SASA has been calculated using $1.4 \AA$ as the effective radius of an atom with VMD, and the constants $\gamma$ and $a$ were set to $0.005 \mathrm{kcalmol}^{-1} \AA^{-2}$ and 0.0 respectively ${ }^{103}$. The entropic term $(T \Delta S)$ can be estimated by a normal-mode analysis of the vibrational frequencies ${ }^{104,105}$. However we have ignored the entropy contribution owing to the translational, rotational and vibrational degrees of freedom in our calculation due to its high computational demand. Finally the aggregation process proceeds via the counter-play between three types of contributions $\left(\Delta G_{\text {elec }}, \Delta G_{v d w}, \Delta G_{\text {solv }}\right)$ of $\Delta G_{\text {bind }}$.

Figure 10(a) shows the evolution of electrostatic interaction energy calculated for the aggregates including the condensed ions around aggregates. The contributions originating from electrostatic interaction reveal that for $\mathrm{NaCl}$ salt solution systems, the net electrostatic energy is attractive in nature despite the presence of negatively charged Glu residue in each the peptides which should result in repulsive interaction between the peptides. The electrostatic attractive interaction between the salt-cations and Glu residue initiates the aggregation events by suppressing the effect of thermodynamically unfavourable electrostatic repulsive interaction between peptides. As noted before, for $\mathrm{NaCl}$ solution systems, a relatively higher number of $\mathrm{Na}^{+}$ions are buried within the peptide aggregates compared to both $\mathrm{MgCl}_{2}$ and $\mathrm{KCl}$ solution systems. The snapshots of buried alkali-ions (monovalent) within the aggregates at the end of 100ns simulation are shown in Figure 10(b-c). The buried cations effectively renormalize the overall charge of the peptides, albeit in different ways (see next section), resulting in effective attractive interaction energies between peptides (in addition to short range hydrophobic energies).

In Figure 9 the number of $\mathrm{Na}^{+}$ions bound to Glu was found to be increased with time indicating the cumulative nature of process, i.e. the propensity of Glu residue on the surface of aggregates to imbibe more $\mathrm{Na}^{+}$ ions from bulk water as the aggregate size increased and in effect this minimize the surface charge density which in turn further assists the growth of aggregates by weakening the effect of negative charge of peptides. Due to the less propensity of $\mathrm{K}^{+}$ions to interact with Glu, $\mathrm{K}^{+}$ions could not compensate the electrostatic repulsive energy of peptide molecules and cause small aggregates formation instead of a single large aggregate. Also, in Figure 10(c)-(d) the decrease in solvation energy term with time and increase in vdW energy with time respectively favor the aggregation process for all cases. We notice vdW energy is more negative for $\mathrm{NaCl}$ case than $\mathrm{KCl}$ case and that is because of larger aggregate size. As there is no significant interaction between the $\mathrm{Mg}^{2+}$ ions and charged peptide molecules, the $\Delta G_{\text {solv }}$ contribution originating from hydrophobic interaction between the peptide molecules is the main driving force 

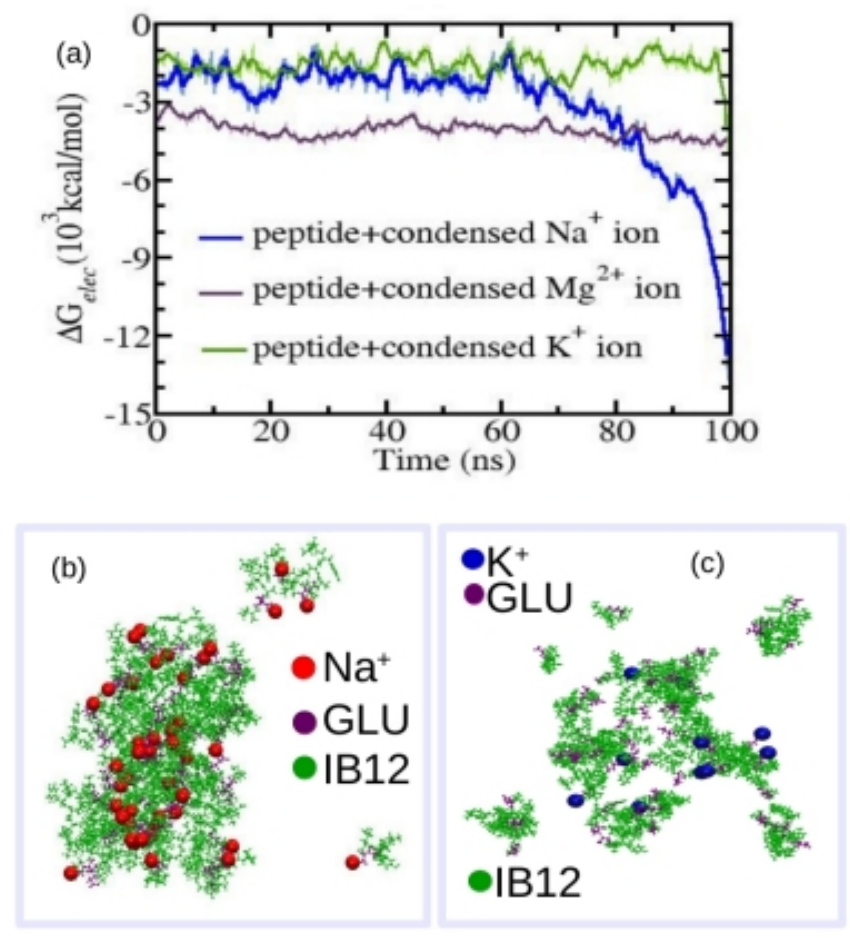
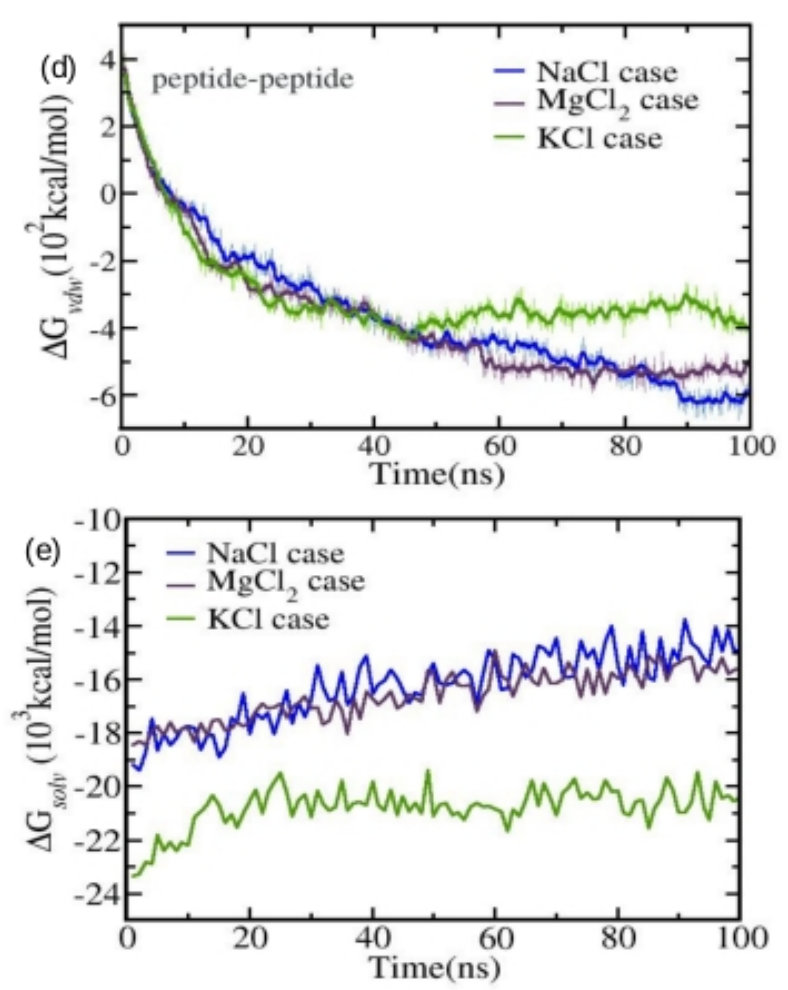

FIG. 10. (a) Time-dependent comparison of electrostatic interaction energies for peptide aggregates and ions bound to peptide for three different IB12-salt systems. (b)-(c) Snapshots of buried alkali ions of salt within the IB12 peptide aggregates in salt solution captured at the end of $100 \mathrm{~ns}$ simulation. The color code red, blue, purple and green present the $\mathrm{Na}^{+}, \mathrm{K}^{+}$, residue-Glu and peptide atoms (in CPK representation) respectively. Water molecules and $\mathrm{Cl}^{-}$ions are omitted for clarity. (d) VDW interaction energy between the peptide atoms for three different IB12- salt systems. (e) Solvation free energy ( $\left.\mathrm{G}_{\text {solv }}\right)$ of peptide molecules originating from the interaction btween water and peptide atoms.

for preferential association of charged polypeptide in the presence of $\mathrm{MgCl}_{2}$ salt. Importantly, the electrostatic interaction of $\mathrm{Na}^{+}$-Glu and hydrophobic interaction both make favorable contributions to the formation of the large aggregates in $\mathrm{NaCl}$-salt solution while unfavorable interpeptide electrostatic interaction and the solvation effect results the formation of small sized aggregates in $\mathrm{KCl}$-salt solution.

\section{E. Charge renormalization of aggregates by different cations}

It is clear from the above section that the final aggregate sizes are the result of the balance of three types of competing interactions namely protein-ion, ion-pair and water-ion interaction. This suggests that at certain values of aggregate sizes, the polypeptide system can achieve minimum energy and get stablized via the resultant interaction energy and results in discretization of aggregate sizes as obtained in the Figure 2. In this section we explore the effective renormalization of the peptide charge by the inclusion of salt, as compared to the control case, to understand the faster aggregation rates, in general, for salt-solutions compared to control system. Previous MD studies and electrophoretic measurements of the binding of divalent cations to insulin in aqueous salt solutions ${ }^{106}$ reported a significant reduction of the absolute value of the electrophoretic mobility of charged insulin in the presence of divalent alkali cations via charge rescaling. Previous works have also shown that multivalent ions can lead to overcompensation of charges, in addition to charge neutralization, and this can be a mode of aggregation of charged polymers such as DNA in the presence of multivalent ions ${ }^{73-75}$.

In Figure 11, a comparison of the effective charge of each peptide as a function of distance $(r)$ from the peptide for the three IB12-salt solution and control system is shown. A striking feature of the plot is that compared to the control system, the effective charge is renormalized at much shorter distances in the presence of salt, though there are significant differences between the salts themselves. This strongly underscores the faster kinetics of aggregation formation of the charged peptides in the presence of excess salts. However, significant differences 


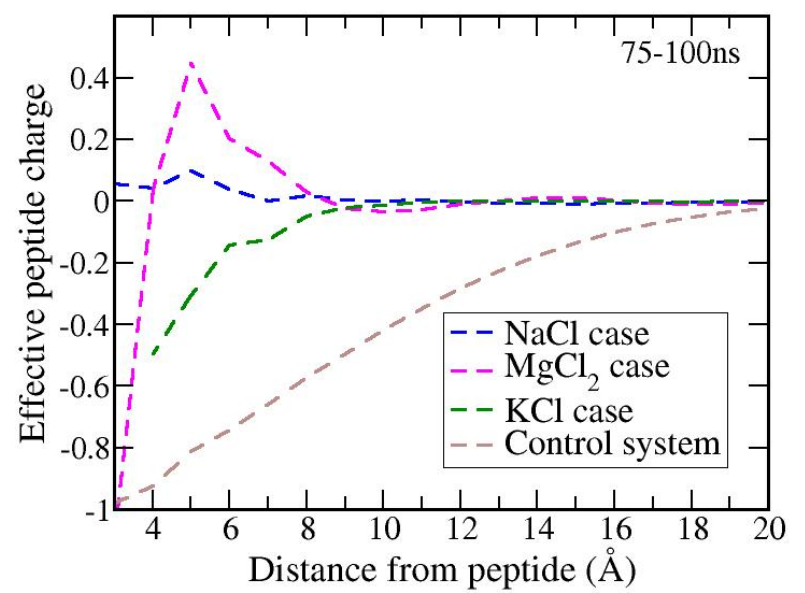

FIG. 11. Effective charge of each peptide as a function of the distance from the peptide heavy atoms (i.e. sum of the net peptide charge together with the charge of all ions located within a given distance of the peptide averaged over all 64 peptides), as obtained from last $25 \mathrm{~ns}$ simulation with $\mathrm{NaCl}$ (blue), $\mathrm{MgCl}_{2}$ (magenta), $\mathrm{KCl}$ (green) and control system (brown).

do exist among the charge renormalization behavior between the three salts at very short distances, which can be the reason behind different sizes and stabilities of the aggregates for the three salt solutions. For $\mathrm{NaCl}$ case, it can be seen that the charge neutralization is achieved at a very small distance from the peptide and this underpins the fastest and the most abundant aggregate size formation for $\mathrm{NaCl}$ salt solution. For both $\mathrm{KCl}$ and $\mathrm{MgCl}_{2}$ salt solutions, the analysis strongly suggests that the cations are not as effective as the Na cations in renormalizing the charges at short distances. Infact for $\mathrm{Mg}^{2+}$ ions, a charge inversion is clearly observed, where the net effective charge changes sign and this is consistent with previous studies on effects on multivalent cations on charged biopolymers such as DNA and other macroions ${ }^{42,73}$. In our analysis we also find that $\mathrm{Mg}^{2+}$ ions are prone to bound with $\mathrm{Cl}^{-}$ions in water largely. Therefore, the polarization field of $\mathrm{MgCl}_{2}$ in water may be the cause of the observed charge inversion of charged peptide and that in turn may effect the aggregation rate. Indeed, for $\mathrm{MgCl}_{2}$ salt $\mathrm{Mg}^{2+}$ ions accumulate in the spherically symmetric surface only untill the point of neutrality is reached. Also, for $\mathrm{MgCl}_{2}$ salt, the electrostatic shielding effect of excessive number of $\mathrm{Cl}^{-}$ions may cause a hinderance for $\mathrm{Mg}^{2+}$ ions to come close to peptide atoms.

\section{F. Condensed ion lifetime distribution}

Solvated ions have important functional roles in influencing the structure and dynamics of biomolecules ${ }^{48-54}$. In this context, lifetime measurement of ions i.e. the res- idence time of ions in the vicinity of peptide chains is an important tool in our understanding of the ion-protein interaction and subsequent insight into the effect of salt on the aggregation process of multipeptide system. Here we calculate lifetimes of condensed ions and report the distribution of the same as shown in Figure 12. An ion is considered to be a condensed ion, if it is at a distance $<=3 \AA$ from any of the peptide atom. Figure 12 shows the distribution of lifetime on a log-log scale for cations and anions of salt separately. In Figure 12(a) we observe

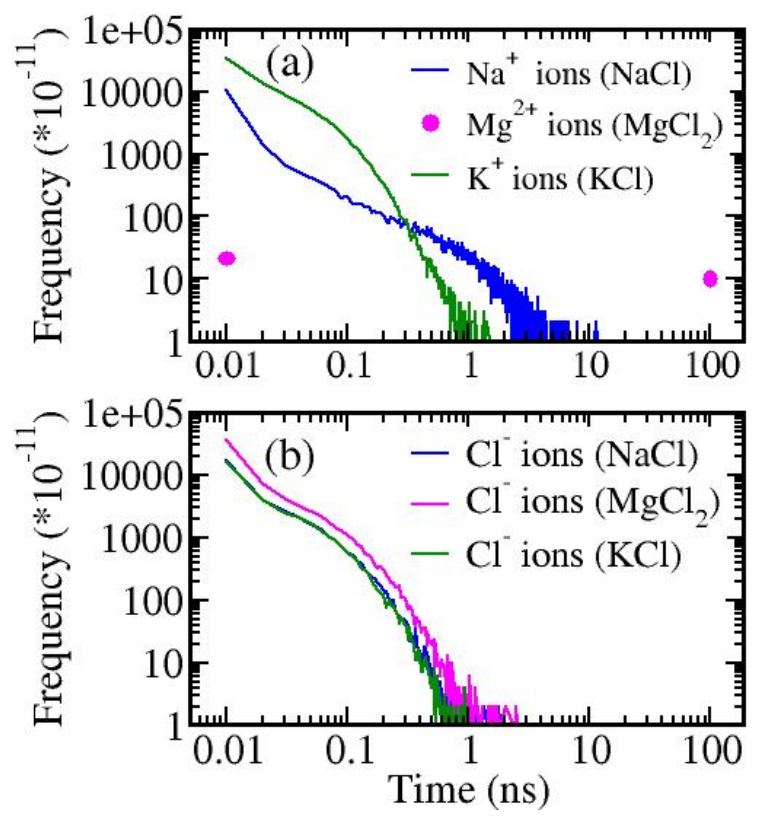

FIG. 12. Historgram of condensed ion lifetime (i.e. residence time of an ion within $3 \AA$ of the backbone atoms of proteins) plotted on a double-log scale for cations(a) and anions(b) separately in three different salt solutions.

a change in the slope in the life time distribution plot of condensed cations, obtained from 100ns long IB12 trajectories, before and after 100ps time scale. The slope of frequency vs. time is nearly constant at the trailing part of the curve albeit small fluctuations. Since there may always be few ions which are likely to interact with peptides at any point of time, the frequency of lifetime distribution for such ions lies in region between 0-100ps of the timescale which is relaxation timescale of the solvated system. The interaction of ions with lifetime less than 100ps are removed from our consideration due to their strong interaction. The plot with greater than 100 ps timescale indicates power-law behavior. When slope of the plot is high, the number of condensed ions with large lifetime is smaller than the number of condensed ions with low lifetime. In Figure 12(b) We don't observe any significant difference in the slope of the lifetime distribution curve of condensed $\mathrm{Cl}^{-}$ions for three types of salts, only difference is the increased frequency value of condensed $\mathrm{Cl}^{-}$ions for divalent salt compared 
to monovalent because of higher number of $\mathrm{Cl}^{-}$ions in simulation box in the case of $\mathrm{MgCl}_{2}$. Therefore, we can say that salt-anions don't have important role in aggregation process of negatively charged IB12-salt system due to electrostatic repulsion between $\mathrm{Cl}^{-}$ion and amino acid Glu residue. In the case of condensed cations the slopes of the histogram plot are different for three different types of salt. The slope of histogram plot of lifetime is less steeper for $\mathrm{NaCl}$ case than $\mathrm{KCl}$ and the residence time of +ve condensed ion around peptide atoms extends upto $10 \mathrm{~ns}$ and $1.5 \mathrm{~ns}$ for $\mathrm{Na}^{+}$and $\mathrm{K}^{+}$ions respectively, suggesting the higher residence times, hence higher interaction strength of $\mathrm{Na}^{+}$ions with peptides than for $\mathrm{K}^{+}$ions. In case of $\mathrm{MgCl}_{2}$, the number of $\mathrm{Mg}^{2+}$ ions near the peptides is small, and the initial $\mathrm{Mg}^{2+}$ ions get trapped within the aggregate at the very early stage of simulation and remain attached to the surface of amino acid Glu throughout the simulation and this results in a single data point near the 100ns time scale on the plot. On average, the $\mathrm{Na}^{+}$ions show significantly greater probability to be bound with negatively charged polypeptide molecules in peptide-salt solution compared to other two cations.

\section{G. MSM analysis of Aggregate size distribution}

Figure 13(a)-(b) shows the evolution of fractional populations of $N$-mer aaggregates with propagation step $(n)$ for top ten high-populated aggregate states in the case of $\mathrm{NaCl}$ and $\mathrm{MgCl}_{2}$ respectively computed using MSM analysis. The components of the transition probability matrix $(P)$ were calculated as the fraction of $i$-mers undergoing a transition to a $j$-mer at a given propagation step. We observe that the system starts to equilibrate after $75 \mathrm{~ns}$, hence in our MSM analysis we used the total 100ns of two MD trajectories for each case for constructing the transition probability matrix.

MSM analysis for $\mathrm{NaCl}$ salt revealed that the top ten highly populated amorphous aggregate states are 52,53 , $54,51,55,50,58,56,57,40-$ mer and the probability of these aggregate sizes increased with time and saturates after a certain propagation step. The aggregate of size 52 has the highest possibility to be formed for a 64 mer IB12 system with $\mathrm{NaCl}$ salt and the probability of higher order aggregate is decreased with size of aggregate. For the medium size cluster of size 40 we observe a slight bump on the occupancy of $N$-mer aggregates with a decrease in fraction indicating that the medium clusters were growing to larger amorphous aggregates $(>$ 50 -mer). However, in the case of $\mathrm{MgCl}_{2}$ salt, the smaller and medium size aggregates have higher fraction in the trajectory. Initially the small size aggregates (4-mer, 5mer, 6-mer, 18-mer) have large fraction and they rapidly accumulate to medium size clusters (32-mer, 33-mer, 42mer, 43-mer). The fraction of medium aggregates consol-

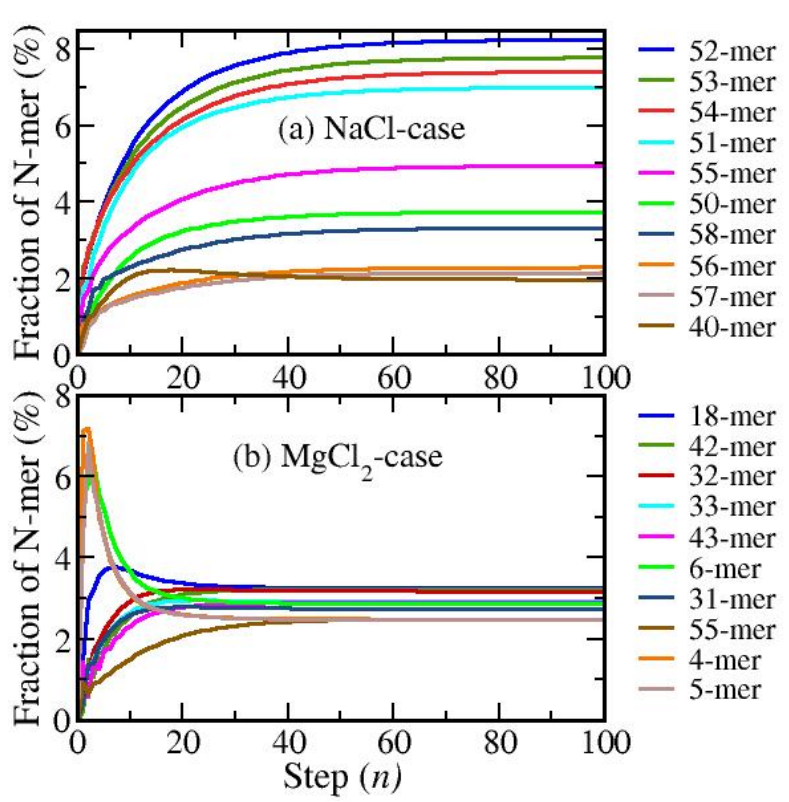

FIG. 13. Markov State Model analysis of $N$-mer fraction for top ten higher populated aggregate states for $\mathrm{NaCl}(\mathrm{a})$ and $\mathrm{MgCl}_{2}$ (b) salt solutions, computed by solving master equation.

idates with propagation step but the formation of large aggregates was found to be less probable for $\mathrm{MgCl}_{2}$ salt solution compared to $\mathrm{NaCl}$ salt solution. This strongly suggests that at the initial stage of aggregation pathway, the smaller size clusters play key role in the formation of larger clusters and different salts can effect this differently.

\section{DISCUSSION AND CONCLUSION}

In this article, a systematic analysis of the effect of salt at high concentration on the amorphous aggregation of charged peptides (IB12) in water have been reported using MD simulations and detailed analyses involving MM/GBSA and charge renormaliztion by salt-ions. Two independent 100ns MD simulations with two monovalent salts $(\mathrm{NaCl}$ and $\mathrm{KCl})$ and one divalent salt $\left(\mathrm{MgCl}_{2}\right)$ corresponding to IB12-salt solutions, and a control simulation with no additional salt (except to achieve charge neutrality) are performed. Efforts have been made to obtain an understanding of the aggregate morphology of the multi-peptide system and the effects of not only the valency of the added salt cations, but also type of cation as well.

The results of this study strongly suggest that the presence $\mathrm{NaCl}$ salt in solution leads to the formation of largest aggregate of 64-IB12 peptides, compared to other salt systems studied, including another monovalent salt $\mathrm{KCl}$. For $\mathrm{MgCl}_{2}$ salt, the simulations suggest 
that though the propensity to form large aggregates is higher than $\mathrm{KCl}$, the stability of formed aggregates is weaker than that of $\mathrm{NaCl}$ salt systems. Possible reasons for this include the cation-peptide interactions, cation size, competing favourable electrostatic and hydrophobic interactions and effective charge renormalization capabilities of the cations considered. The simulations suggest that the formation of large aggregate in $\mathrm{NaCl}$-salt solution originated from both attractive electrostatic interaction of $\mathrm{Na}^{+}$-Glu (negatively charged) and subsequent hydrophobic interactions between amino acids of peptide. However, in the case of $\mathrm{MgCl}_{2}$-salt solution, the predominant favourable interactions in aggregate formation are hydrophobic in nature.

Charge renormalization by the counterions has been suggested to be one of the important pathways of aggregation of highly charged polymer systems ${ }^{73-75}$. In these systems, the condensed counterions effectively render the similarly charged polymers neutral, thereby inducing aggregation via short-range attractive interactions ${ }^{74}$. The effective charge of each peptide (averaged over 64 peptides) as a function of distance from the peptide is measured and it shows strong indications that the presence of $\mathrm{NaCl}$ effectively neutralizes the system, which is not the case for the other two salt solutions considered in the study. Significantly, the absolute value of effective peptide charge is positive for $\mathrm{MgCl}_{2}$ case and indeed, the overscreening of peptide charge by the $\mathrm{Mg}^{2+}$ cloud around the aggregates drives the peptide aggregation in $\mathrm{MgCl}_{2}$ solutions. So it is worth to conclude that though final aggregate sizes for $\mathrm{NaCl}$ and $\mathrm{MgCl}_{2}$ are comparable, the mechanism of aggregate formation is fundamentally different for the two cases. In case of other monovalent salt, $\mathrm{KCl}$, the smaller and unstable aggregate formation is due to the lack of interaction between $\mathrm{K}^{+}$and Glu residue of the IB12 peptides. This leads to unfavorable inter-peptide electrostatic repulsive interactions, which possibly cannot be compensated by the favourable inter hydrophobic interactions and thereby results in smaller and more unstable aggregates. The calculation of lifetime of condensed ions also revealed that $\mathrm{Na}^{+}$ ions have much more propensity to be bound to the aggregates for much longer time than $\mathrm{K}^{+}$ions. Markov state model analysis suggested that small aggregates consisting of $4 \sim 6$ peptides accumulate at initial stages of aggregation and then they merge into large aggregates. Interestingly, our simulations for IB12 peptide in explicit solvent environment using recent all-atom CHARMM36 force field have displayed precipitation of peptide monomers into large amorphous aggregate state but do not show significant conformational conversion of soluble peptides into insoluble amyloid-like fibrils contrary to expectations from previous simulations ${ }^{59,60}$. It has also been recently suggested that the force field may play a role in elucidating the final conformations of such peptides in solution ${ }^{107}$. Therefore, caution is needed to chose appropriate force-field parameters in the protein aggregation studies. However, the primary focus of this study is not on the fibrillar formation of either single or multiple aggregates, but to understand the role of salts on the aggregation dynamics of charged peptides in solutions. Much longer simulations and multiple simulations are most likely required for detailed understanding of the effect of different force fields and their inherent propensity for certain secondary structure formations, which is beyond the scope of the present study.

\section{SUPPLEMENTARY MATERIAL}

See supplementary material for the normalized aggregate size distribution of charged poly-peptide system in $\mathrm{NaCl}$ and $\mathrm{MgCl}_{2}$ salt solution with TIP4P-Ew water model, the $\mathrm{g}(\mathrm{r})$ function for ion-pair and water-ion interactions, and snapshots of condensed anions and water molecules around a single cation.

Acknowledgments: The simulations were carried out on the supercomputing machines Annapurna and Nandadevi at the Institute of Mathematical Sciences.

\section{REFERENCES}

${ }^{1}$ C. A. Ross and M. A. Poirier, Nature medicine 10, S10 (2004).

${ }^{2}$ A. J. Espay, J. A. Vizcarra, L. Marsili, A. E. Lang, D. K. Simon, A. Merola, K. A. Josephs, A. Fasano, F. Morgante, R. Savica, et al., Neurology 92, 329 (2019).

${ }^{3}$ W. Wang, S. Nema, and D. Teagarden, International journal of pharmaceutics 390, 89 (2010).

${ }^{4}$ E. Y. Chi, S. Krishnan, T. W. Randolph, and J. F. Carpenter, Pharmaceutical research 20, 1325 (2003).

${ }^{5}$ A. Sharma, J. M. Pasha, and S. Deep, Journal of colloid and interface science 350, 240 (2010).

${ }^{6}$ C. Haass and D. J. Selkoe, Nature reviews Molecular cell biology 8, 101 (2007).

${ }^{7}$ R. Riek, P. Güntert, H. Döbeli, B. Wipf, and K. Wüthrich, European journal of biochemistry 268, 5930 (2001).

${ }^{8}$ B. Caughey and P. T. Lansbury Jr, Annual review of neuroscience 26, 267 (2003).

${ }^{9}$ T. Eichner and S. E. Radford, The FEBS journal 278, 3868 (2011).

${ }^{10}$ K. M. Lundberg, C. J. Stenland, F. E. Cohen, S. B. Prusiner, and G. L. Millhauser, Chemistry \& biology 4, 345 (1997).

${ }^{11}$ D. M. Walsh, I. Klyubin, J. V. Fadeeva, W. K. Cullen, R. Anwyl, M. S. Wolfe, M. J. Rowan, and D. J. Selkoe, Nature 416, 535 (2002).

${ }^{12}$ E. W. Martin and T. Mittag, Biochemistry 57, 2478 (2018). 
${ }^{13}$ M. Kato, T. W. Han, S. Xie, K. Shi, X. Du, L. C. Wu, H. Mirzaei, E. J. Goldsmith, J. Longgood, J. Pei, et al., Cell 149, 753 (2012).

${ }^{14}$ T. J. Nott, E. Petsalaki, P. Farber, D. Jervis, E. Fussner, A. Plochowietz, T. D. Craggs, D. P. Bazett-Jones, T. Pawson, J. D. Forman-Kay, et al., Molecular cell 57, 936 (2015).

${ }^{15}$ S. Elbaum-Garfinkle, Y. Kim, K. Szczepaniak, C. C.H. Chen, C. R. Eckmann, S. Myong, and C. P. Brangwynne, Proceedings of the National Academy of Sciences 112, 7189 (2015).

${ }^{16}$ Y. Lin, S. L. Currie, and M. K. Rosen, Journal of Biological Chemistry 292, 19110 (2017).

${ }^{17}$ F. G. Quiroz and A. Chilkoti, Nature materials 14, 1164 (2015).

${ }^{18}$ A. Molliex, J. Temirov, J. Lee, M. Coughlin, A. P. Kanagaraj, H. J. Kim, T. Mittag, and J. P. Taylor, Cell 163, 123 (2015).

${ }^{19}$ S. C. Weber, Current Opinion in Cell Biology 46, 62 (2017), cell Nucleus.

${ }^{20}$ C. W. Pak, M. Kosno, A. S. Holehouse, S. B. Padrick, A. Mittal, R. Ali, A. A. Yunus, D. R. Liu, R. V. Pappu, and M. K. Rosen, Molecular cell 63, 72 (2016).

${ }^{21}$ T. Murakami, S. Qamar, J. Q. Lin, G. S. K. Schierle, E. Rees, A. Miyashita, A. R. Costa, R. B. Dodd, F. T. Chan, C. H. Michel, et al., Neuron 88, 678 (2015).

${ }^{22}$ Y. Lin, D. S. Protter, M. K. Rosen, and R. Parker, Molecular cell 60, 208 (2015).

${ }^{23}$ T. Niwa, B.-W. Ying, K. Saito, W. Jin, S. Takada, T. Ueda, and H. Taguchi, Proceedings of the National Academy of Sciences 106, 4201 (2009).

${ }^{24}$ M. S. Lawrence, K. J. Phillips, and D. R. Liu, Journal of the American Chemical Society 129, 10110 (2007).

${ }^{25} \mathrm{P}$. Simeonov, R. Berger-Hoffmann, R. Hoffmann, N. Sträter, and T. Zuchner, Protein Engineering, Design \& Selection 24, 261 (2010).

${ }^{26}$ G. S. Manning, J. Chem. Phys. 51, 924 (1969).

${ }^{27}$ M. Deserno and C. Holm, Mol. Phys. 100, 2941 (2002).

${ }^{28}$ S. M. Mel'nikov, M. O. Khan, B. Lindman, and B. Jönsson, J. Am. Chem. Soc. 121, 1130 (1999).

${ }^{29}$ A. V. Dobrynin and M. Rubinstein, Prog. Poly. Science 30, 1049 (2005).

${ }^{30}$ R. R. Netz and D. Andelman, Phys. Rep. 380, 1 (2003).

${ }^{31}$ P. J. Flory, Principles of Polymer Chemistry (Cornell University, Ithaca, 1953).

${ }^{32}$ A. Y. Grosberg and A. R. Khokhlov, Statistical Physics of Macromolecules (AIP Press, Woodbury, NY, 1994).

${ }^{33}$ P. Kundu and A. Dua, J. Stat. Mech. , P07023 (2014).

${ }^{34}$ H. Schiessel and P. Pincus, Macromolecules 31, 7953 (1998).

${ }^{35}$ R. G. Winkler, M. Gold, and P. Reineker, Phys. Rev. Lett. 80, 3731 (1998).

${ }^{36}$ H. J. Limbach*, , and C. Holm, J. Phys. Chem. B 107, 8041 (2003), http://dx.doi.org/10.1021/jp027606p.

${ }^{37}$ A. M. Tom, R. Rajesh, and S. Vemparala, J. Chem.
Phys. 144, 034904 (2016).

${ }^{38}$ A. Varghese, R. Rajesh, and S. Vemparala, J. Chem. Phys. 137, 234901 (2012).

${ }^{39}$ A. Varghese, S. Vemparala, and R. Rajesh, J. Chem. Phys. 135, 154902 (2011).

${ }^{40}$ A. M. Tom, S. Vemparala, R. Rajesh, and N. V. Brilliantov, Physical review letters 117, 147801 (2016).

${ }^{41}$ A. M. Tom, R. Rajesh, and S. Vemparala, The Journal of chemical physics 147, 144903 (2017).

${ }^{42}$ T. T. Nguyen, A. Y. Grosberg, and B. I. Shklovskii, Physical review letters 85, 1568 (2000).

${ }^{43}$ M. Muthukumar, J. Chem. Phys. 120, 9343 (2004).

${ }^{44}$ A. A. Gavrilov, A. V. Chertovich, and E. Y. Kramarenko, Macromolecules 49, 11031110 (2016).

${ }^{45}$ E. Y. Kramarenko, A. R. Khokhlov, and K. Yoshikawa, Macromol. Theory Simul. 9, 249 (2000).

${ }^{46}$ F. J. Solis and O. de la Cruz, J. Chem. Phys. 112, 2030 (2000).

${ }^{47}$ A. R. Khokhlov and E. Y. Kramarenko, Macromolecules 29, 681 (1996).

${ }^{48}$ S. Jain and J. B. Udgaonkar, Biochemistry 49, 7615 (2010).

${ }^{49}$ P. Gupta and S. Deep, RSC Advances 5, 95717 (2015).

${ }^{50}$ J. Zhang, in Protein-Protein InteractionsComputational and Experimental Tools (IntechOpen, 2012).

${ }^{51}$ K. Tsumoto, D. Ejima, A. M. Senczuk, Y. Kita, and T. Arakawa, Journal of pharmaceutical sciences 96, 1677 (2007).

${ }^{52}$ K. Klement, K. Wieligmann, J. Meinhardt, P. Hortschansky, W. Richter, and M. Fändrich, Journal of molecular biology 373, 1321 (2007).

${ }^{53}$ V. Yeh, J. M. Broering, A. Romanyuk, B. Chen, Y. O. Chernoff, and A. S. Bommarius, Protein Science 19, 47 (2010).

${ }^{54}$ P. Arosio, B. Jaquet, H. Wu, and M. Morbidelli, Biophysical chemistry 168, 19 (2012).

${ }^{55}$ B. Raman, E. Chatani, M. Kihara, T. Ban, M. Sakai, K. Hasegawa, H. Naiki, C. M. Rao, and Y. Goto, Biochemistry 44, 1288 (2005).

${ }^{56}$ M. Bostrom, D. F. Parsons, A. Salis, B. W. Ninham, and M. Monduzzi, Langmuir 27, 9504 (2011).

${ }^{57}$ A. M. Ruschak and A. D. Miranker, Journal of molecular biology 393, 214 (2009).

${ }^{58}$ P. C. van der Wel, J. R. Lewandowski, and R. G. Griffin, Biochemistry 49, 9457 (2010).

${ }^{59}$ D. Matthes, V. Gapsys, V. Daebel, and B. L. De Groot, PLoS One 6, e19129 (2011).

${ }^{60}$ D. Matthes, V. Gapsys, and B. L. de Groot, Journal of molecular biology 421, 390 (2012).

${ }^{61}$ J. S. Sharp, J. A. Forrest, and R. A. L. Jones, Biochemistry 41, 15810 (2002).

${ }^{62}$ L. Nielsen, R. Khurana, A. Coats, S. Frokjaer, J. Brange, S. Vyas, V. N. Uversky, and A. L. Fink, Biochemistry 40, 6036 (2001).

${ }^{63}$ F. Collu, E. Spiga, N. Chakroun, H. Rezaei, and F. Fraternali, Chemical Communications 54, 8007 
(2018).

${ }^{64}$ N. Cerda-Costa, A. Esteras-Chopo, F. Aviles, L. Serrano, and V. Villegas, Journal of molecular biology 366, 1351 (2007).

${ }^{65} \mathrm{~S}$. Genheden and U. Ryde, Expert opinion on drug discovery 10, 449 (2015).

${ }^{66} \mathrm{P}$. Tuffery and P. Derreumaux, Journal of The Royal Society Interface 9, 20 (2011).

${ }^{67}$ C. R. Schwantes, R. T. McGibbon, and V. S. Pande, The Journal of chemical physics 141, 09B201_1 (2014).

${ }^{68}$ V. S. Pande, K. Beauchamp, and G. R. Bowman, Methods 52, 99 (2010).

${ }^{69}$ M. Senne, B. Trendelkamp-Schroer, A. S. Mey, C. Schutte, and F. Noe, Journal of chemical theory and computation 8, 2223 (2012).

${ }^{70}$ N.-V. Buchete and G. Hummer, The Journal of Physical Chemistry B 112, 6057 (2008).

${ }^{71}$ C. Schütte, F. Noé, J. Lu, M. Sarich, and E. VandenEijnden, The Journal of chemical physics 134, 05B609 (2011).

${ }^{72}$ V. S. Pande, in An introduction to Markov state models and their application to long timescale molecular simulation (Springer, 2014) pp. 101-106.

${ }^{73}$ I. Borukhov, K.-C. Lee, R. F. Bruinsma, W. M. Gelbart, A. J. Liu, and M. J. Stevens, The Journal of chemical physics 117, 462 (2002).

${ }^{74}$ F. Zhang, M. Skoda, R. Jacobs, S. Zorn, R. A. Martin, C. Martin, G. Clark, S. Weggler, A. Hildebrandt, O. Kohlbacher, et al., Physical review letters 101, 148101 (2008).

${ }^{75}$ E. Trizac, L. Bocquet, and M. Aubouy, Physical review letters 89, 248301 (2002).

${ }^{76}$ W. L. Jorgensen, J. Chandrasekhar, J. D. Madura, R. W. Impey, and M. L. Klein, The Journal of Chemical Physics 79, 926 (1983).

${ }^{77}$ H. W. Horn, W. C. Swope, J. W. Pitera, J. D. Madura, T. J. Dick, G. L. Hura, and T. Head-Gordon, The Journal of Chemical Physics 120, 9665 (2004).

${ }^{78}$ A. D. MacKerell, D. Bashford, M. Bellott, R. L. Dunbrack, J. D. Evanseck, M. J. Field, S. Fischer, J. Gao, H. Guo, S. Ha, D. Joseph-McCarthy, L. Kuchnir, K. Kuczera, F. T. K. Lau, C. Mattos, S. Michnick, T. Ngo, D. T. Nguyen, B. Prodhom, W. E. Reiher, B. Roux, M. Schlenkrich, J. C. Smith, R. Stote, J. Straub, M. Watanabe, J. WirkiewiczKuczera, D. Yin, and M. Karplus, The Journal of Physical Chemistry B 102, 3586 (1998).

${ }^{79}$ P. Li, B. P. Roberts, D. K. Chakravorty, and K. M. Merz, Journal of Chemical Theory and Computation 9, 2733 (2013).

${ }^{80}$ I. S. Joung and T. E. Cheatham, The Journal of Physical Chemistry B 112, 9020 (2008).

${ }^{81}$ W. Humphrey, A. Dalke, and K. Schulten, Journal of Molecular Graphics 14, 33 (1996).

${ }^{82}$ J. C. Phillips, R. Braun, W. Wang, J. Gumbart, E. Tajkhorshid, E. Villa, C. Chipot, R. D. Skeel, L. Kal, and K. Schulten, Journal of Computational Chemistry 26, 1781 (2005).
${ }^{83} \mathrm{~J}$. Huang and A. D. MacKerell Jr, Journal of computational chemistry 34, 2135 (2013).

${ }^{84}$ S. E. Feller, Y. Zhang, R. W. Pastor, and B. R. Brooks, The Journal of chemical physics 103, 4613 (1995).

${ }^{85}$ T. Darden, D. York, and L. Pedersen, The Journal of chemical physics 98, 10089 (1993).

${ }^{86}$ M. Sayar and C. Holm, EPL (Europhysics Letters) 77, 16001 (2006).

${ }^{87}$ H. Fazli, S. Mohammadinejad, and R. Golestanian, Journal of Physics: Condensed Matter 21, 424111 (2009).

${ }^{88}$ H. Fazli and R. Golestanian, Physical Review E 76, 041801 (2007).

${ }^{89}$ M. Sayar and C. Holm, Physical Review E 82, 031901 (2010).

${ }^{90}$ A. Zhu, S. Dai, L. Li, and F. Zhao, Colloids and Surfaces B: Biointerfaces 47, 20 (2006).

${ }^{91} \mathrm{~T}$. Ghosh, A. Kalra, and S. Garde, The Journal of Physical Chemistry B 109, 642 (2005).

${ }^{92}$ T. Vicsek and F. Family, Physical Review Letters 52, 1669 (1984).

${ }^{93} \mathrm{P}$. Meakin, Fractals, scaling and growth far from equilibrium, Vol. 5 (Cambridge university press, 1998).

${ }^{94}$ T. P. Light, K. M. Corbett, M. A. Metrick, and G. MacDonald, Langmuir 32, 1360 (2016).

${ }^{95}$ W. Kunz, J. Henle, and B. W. Ninham, Current Opinion in Colloid \& Interface Science 9, 19 (2004).

${ }^{96}$ Y. Zhang and P. S. Cremer, Annual review of physical chemistry 61, 63 (2010).

${ }^{97}$ H. I. Okur, J. Kherb, and P. S. Cremer, Journal of the American Chemical Society 135, 5062 (2013).

${ }^{98}$ M. Mantina, A. C. Chamberlin, R. Valero, C. J. Cramer, and D. G. Truhlar, The Journal of Physical Chemistry A 113, 5806 (2009).

${ }^{99}$ C. J. Roberts, Current opinion in biotechnology 30, 211 (2014).

${ }^{100}$ P. A. Kollman, I. Massova, C. Reyes, B. Kuhn, S. Huo, L. Chong, M. Lee, T. Lee, Y. Duan, W. Wang, et al., Accounts of chemical research 33, 889 (2000).

${ }^{101}$ R. B. Hermann, The Journal of Physical Chemistry 76, 2754 (1972).

${ }^{102}$ G. Amidon, S. Yalkowsky, S. Anik, and S. Valvani, The Journal of Physical Chemistry 79, 2239 (1975).

${ }^{103}$ D. A. Case, V. Babin, J. Berryman, R. Betz, Q. Cai, D. Cerutti, T. Cheatham Iii, T. Darden, R. Duke, H. Gohlke, et al., (2014).

${ }^{104}$ M. D. A, In Statistical Mechanics $2^{\text {nd }}$ edn. (Sausalito, CA:University Science Books), 2000).

${ }^{105}$ B. R. Brooks, D. Janežič, and M. Karplus, Journal of computational chemistry 16, 1522 (1995).

${ }^{106}$ E. Duboue-Dijon, P. Delcroix, H. Martinez-Seara, J. Hladílkova, P. Coufal, T. Krízek, and P. Jungwirth, The Journal of Physical Chemistry B 122, 5640 (2018).

${ }^{107}$ J. Lincoff, S. Sasmal, and T. Head-Gordon, Biophysical Journal 114, 675a (2018). 